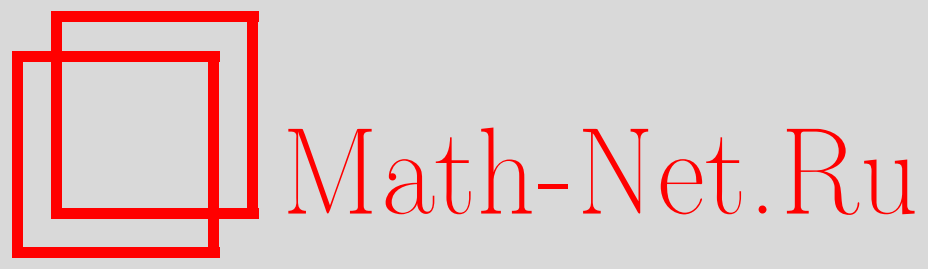

В. А. Сендеров, В. А. Хацкевич, Проблема Кёнигса и крайние неподвижные точки, Функи. анализ и его прил., 2010, том 44, выпуск 1, 87-90

DOI: https://doi.org/10.4213/faa2976

Использование Общероссийского математического портала Math$\mathrm{Net.Ru} \mathrm{подразумевает,} \mathrm{что} \mathrm{вы} \mathrm{прочитали} \mathrm{и} \mathrm{согласны} \mathrm{с} \mathrm{пользователь-}$ ским соглашением

http://www . mathnet.ru/rus/agreement

Параметры загрузки:

IP : 52.6 .47 .48

26 апреля 2023 г., 16:18:37

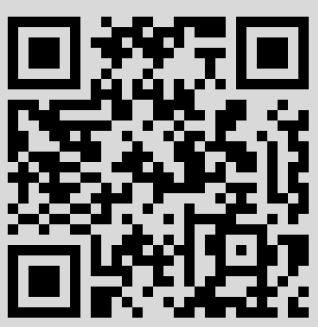


Условие 1) означает, что отображение $T$ является взаимно однозначным и поэтому существует обратное отображение $T^{-1}$, определенное на области значений отображения $T$. Условие 2) означает что область определения обратного отображения $T^{-1}$ содержит конус $K$ (может быть, совпадая с ним). Условие 3 ) означает, что обратное отображение $T^{-1}$ является монотонным.

\title{
ЛитературА
}

[1] М. А. Красносельский, В. Ш. Бурд, Ю. С. Колесов, Нелинейнъе почти периодические колебания, Наука, М., 1970. [2] Ю. В. Трубников, А. И. Перов, Дифференциальные уравнения с монотонными нелинейностями, Наука и техника, Минск, 1986. [3] А. Ф. Тиман, Теория приближения функиий действительного переменного, Физматлит, М., 1960. [4] Ф. Р. Гантмахер, Теория матрии, Наука, М., 1967. [5] М. А. Красносельский, Положительные решения операторных уравнений, Физматлит, М., 1962. [6] Э. Хилле, Р. Филлипс, Функционалъный анализ и полугруппы, ИЛ, М., 1962. [7] И. К. Даугавет, Введение в теорию приближения функиий, Издво ЛГУ, Ленинград, 1977. [8] P. P. Zabrejko, Collect. Math., 48:4-6 (1997), 825-859. [9] Л. Коллатц, Функциональный анализ и вычислительная математика, Мир, М., 1969. [10] Дж. Ортега, В. Рейнболдт, Итерационные методы решения нелинейных систем уравнений со многими неизвестными, Мир, М., 1975. [11] А. И. Перов, Обобщенный принцип сжимающих отображений, Вестник ВГУ, 2005, № 1, 196-207.

Воронежский госуниверситет

e-mail: perov@nosc.vsu.ru

Поступило в редакцию 30 января 2008 г.

УДК $517.432+517.515+515.958$

\section{Проблема Кёнигса и крайние неподвижные точки}

\author{
(c) 2010. В. А. СендЕРов, В. А. ХАцКЕВич
}

Проблема вложения Кёнигса (KE-проблема), возникшая в работах Г. Кёнигса, П. Леви и Ж. Адамара в связи с решением различных прикладных задач, имеет более чем вековую историю. Общая формулировка этой проблемы такова. Пусть $D$ - область комплексного банахова пространства и $f \in \operatorname{Hol}(D)$. Существует ли семейство $\{F(t)\}_{t \geqslant 0} \subset \operatorname{Hol}(D)$, непрерывно (в топологии локально равномерной сходимости над $D$ ) зависящее от $t$ и такое, что $F(0)=I, F(1)=f$ и $F(s+t)=F(s) \circ F(t)$ при всех $s, t \geqslant 0$ ?

Если семейство $\{F(t)\}_{t \geqslant 0}$ существует, то говорят, что $f$ обладает КЕ-свойством.

В последние годы появились новые работы, относящиеся к KЕ-проблеме и к ее приложениям. Так, в [1]-[5] рассматривался случай, когда $D-$ единичный открытый шар пространства $\mathscr{L}\left(\mathfrak{H}_{1}, \mathfrak{H}_{2}\right)$, где $\mathfrak{H}_{1}$ и $\mathfrak{H}_{2}$ - гильбертовы пространства, а $f$ - порождаемое (плюс-)оператором $A$ по формуле (1) (см. ниже) преобразование шара $D$. Этот же случай рассматривается и в данной работе.

Пусть $\mathfrak{H}=\mathfrak{H}_{1} \oplus \mathfrak{H}_{2}-$ комплексное индефинитное $\left(\min \left\{\operatorname{dim} \mathfrak{H}_{1}, \operatorname{dim} \mathfrak{H}_{2}\right\}>0\right)$ пространство Крейна и $A$-оператор с блок-матрицей $\left(\begin{array}{ll}A_{11} & A_{12} \\ A_{21} & A_{22}\end{array}\right)$. Пусть 
$D=\mathscr{K}$ - открытый единичный шар пространства $\mathscr{L}\left(\mathfrak{H}_{1}, \mathfrak{H}_{2}\right)$. Формула

$$
\mathscr{F}_{A}(K)=\left(A_{21}+A_{22} K\right)\left(A_{11}+A_{12} K\right)^{-1},
$$

где $K \in \mathscr{L}\left(\mathfrak{H}_{1}, \mathfrak{H}_{2}\right)$ и $A_{i j} \in \mathscr{L}\left(\mathfrak{H}_{j}, \mathfrak{H}_{i}\right)$ при $i, j=1,2$, определяет в $\mathscr{L}\left(\mathfrak{H}_{1}, \mathfrak{H}_{2}\right)$ дробно-линейное отображение (д.л.о.) $\mathscr{F}_{A}$.

Обозначение $\mathscr{F}_{A}$ мы будем, как правило, сохранять и за естественными сужениями рассматриваемого отображения.

Ниже мы без специальных оговорок будем использовать определения из функционального анализа и теории пространств с индефинитной метрикой (см. фундаментальные монографии [6] и [7]). В частности, всюду определенный линейный ограниченный оператор $T$ в $\mathfrak{H}$ мы будем называть плюс-оператором, если $[x, x] \geqslant 0$ влечет за собой $[T x, T x] \geqslant 0$. Плюс-оператор $T$ называется строгим, если $\inf _{[x, x]=1}[T x, T x]>0$, и бистрогим, если при этом $T^{*}-$ также строгий плюс-оператор. Далее, оператор $A$ с блок-матрицей $\left(\begin{array}{ll}A_{11} & A_{12} \\ A_{21} & A_{22}\end{array}\right)$ мы будем, в соответствии с терминологией работ [8]-[10], называть верхним треугольным, если $A_{21}=0$, и нижним треугольным, если $A_{12}=0$.

Напомним, что крайними точками единичного шара пространства $\mathscr{L}\left(\mathfrak{H}_{1}, \mathfrak{H}_{2}\right)$ являются максимальные частичные изометрии, т. е. изометрии $\left(C: C^{*} C=I\right)$ и коизометрии $\left(C: C C^{*}=I\right)$ [11, гл. XIII, п. 99].

Пусть плюс-оператор $A$ определяет аффинное отображение $\mathscr{F}_{A}$,

$$
\mathscr{F}_{A}(K)=\left(A_{21}+A_{22} K\right) A_{11}^{-1},
$$

открытого шара $\mathscr{K}$ в себя. Очевидно, что $\mathscr{F}_{A}(\overline{\mathscr{K}}) \subseteq \overline{\mathscr{K}}$; кроме того, д.л.о. $\mathscr{F}_{A}: \overline{\mathscr{K}} \rightarrow \overline{\mathscr{K}}$ непрерывно в слабой операторной топологии. Поскольку, помимо этого, непустое выпуклое множество $\overline{\mathscr{K}}$ в этой топологии компактно, то по теореме Шаудера-Тихонова-Гликсберга (см., например, [7, гл. III, теорема 3.6]) отображение $\mathscr{F}_{A}$ имеет в $\overline{\mathscr{K}}$ хотя бы одну неподвижную точку.

Пусть $C$ - неподвижная точка д.л.о. $\mathscr{F} A$ вида (2). Тогда $A_{21}+A_{22} C=C A_{11}$, или $A_{21}=C A_{11}-A_{22} C$. Подставляя последнее равенство в (2), получаем

$$
\mathscr{F}_{A}(K)=A_{22}(K-C) A_{11}^{-1}+C,
$$

где $K \in \mathscr{K}$.

Лемма 1. Пусть $\mathscr{F}_{A}: \mathscr{K} \rightarrow \mathscr{K}-$ д.л.о. вида либо

1) $\mathscr{F}_{A}(K)=A_{22}(K-C)+C$, где $K \in \mathscr{K}, C-$ коизометрия, либо

2) $\mathscr{F}_{A}(K)=(K-C) A_{11}^{-1}+C$, где $K \in \mathscr{K}, C-$ изометрия. Тогда $\sigma_{p}\left(A_{22}\right) \subseteq(0,1]$ в случае 1$), \sigma_{p}\left(A_{11}^{-1}\right) \subseteq(0,1]$ в случае 2.

Теорема 2. В условиях леммы 1 мы имеем $A_{22}=\alpha I$, где $\alpha \in(0,1]$, в случае 1) и $A_{11}^{-1}=\alpha I$, где $\alpha \in(0,1]$, в случае 2$)$.

Доказательство для случая $\mathbf{1}$. Нетрудно доказать, что

$$
\left(A_{22} x, x\right) \neq 0 \text { при } x \neq 0 .
$$

Пусть $\mathscr{P}: \mathfrak{H}_{2} \rightarrow \mathfrak{H}_{2}^{\prime}-$ произвольный двумерный ортопроектор. Достаточно доказать, что $\mathscr{P} A_{22} \mathscr{P}=\alpha I$. Пусть для некоторого $B=\mathscr{P} A_{22} \mathscr{P}$ это не так. Выберем в $\mathfrak{H}_{2}^{\prime}$ ортонормированный базис $\left\{e_{1}, e_{2}\right\}$, векторы которого не являются собственными для $B$. Из $(*)$ следует, что все элементы матрицы $\left(\begin{array}{ll}\alpha & \gamma \\ \beta & \tau\end{array}\right)$ 
оператора $B$ в этом базисе ненулевые. Из (*) следует также, что $\alpha \tau-\gamma \beta \neq 0$. Определим оператор $\widehat{K} \in \mathscr{L}\left(\mathfrak{H}_{2}\right)$ соотношениями $\widehat{K} e_{1}=x e_{1}+z e_{2}$, где $1>x>$ $\left(|\alpha|^{2}-|\gamma|^{2}\right) /\left(|\alpha|^{2}+|\gamma|^{2}\right), z=\alpha(1-x) / \gamma, \widehat{K}\left(e_{1}^{\perp}\right)=\{0\}$. Так как $|x|^{2}+|z|^{2}<1$, то $\|\widehat{K}\|<1$. Пусть $a=\left(A_{22}(\widehat{K}-I)+I\right) e_{1}$. Тогда $a=e_{1}+(\beta x+\tau z-\beta) e_{2}$, поскольку

$$
\left(\begin{array}{ll}
\alpha & \gamma \\
\beta & \tau
\end{array}\right)\left(\begin{array}{ll}
x & 0 \\
z & 0
\end{array}\right)\left(\begin{array}{l}
1 \\
0
\end{array}\right)=\left(\begin{array}{c}
\alpha \\
\beta x+\tau z
\end{array}\right) .
$$

Так как $\alpha \tau \neq \gamma \beta$, то $\beta x+\tau z-\beta \neq 0$.

Таким образом, $\|a\|>1,\left\|\mathscr{F}_{A}(\widehat{K} C)\right\|>1$. Противоречие.

Замечание 3. Как показывают примеры, заменить коизометрию либо изометрию в теореме 2 произвольным линейным оператором с нормой 1 нельзя. С другой стороны, пусть $\mathscr{F}_{A}: \mathscr{K} \rightarrow \mathscr{K}-$ аффинное д.л.о. вида

$$
\mathscr{F}_{A}(K)=\alpha(K-C)+C,
$$

где $K \in \mathscr{K}, \alpha \in \mathbb{C},\|C\|=1$. Тогда $\alpha \in(0,1]$.

Теорема 4. Пусть $\mathscr{F}_{A}: \mathscr{K} \rightarrow \mathscr{K}-$ афбфинное д.л.о. вида (3), где $K \in \mathscr{K}$, $C$ - унитарный оператор, и пусть либо 1) $A_{11}$, либо 2) $A_{22}$ - унитарный оператор. Тогда $A_{22}=\alpha C A_{11} C^{-1}$, где $\alpha \in(0,1]$.

Доказательство, опирающееся на теорему 2, в случае 1) получается с помощью рассмотрения $\psi$, такого, что

$$
\psi(K)=C A_{11}^{-1} C^{-1}\left(A_{21} A_{11}^{-1}+A_{22} K\right),
$$

а в случае 2) - с помощью рассмотрения $\psi$, такого, что

$$
\psi(K)=\left(K A_{11}^{-1}+C-A_{22} C A_{11}^{-1}\right) C^{-1} A_{22} C,
$$

- аффинных д.л.о., переводящих $\mathscr{K}$ в себя и удовлетворяющих условиям 1) и 2) леммы 1 соответственно.

Теорема 5. Пусть верхний треугольный плюс-оператор $A$, такой, что $0 \in \rho\left(A_{11}\right)$, определяет на некотором подмножсестве пространства $\mathscr{L}\left(\mathfrak{H}_{1}, \mathfrak{H}_{2}\right)$ д.л.о. $\mathscr{F}_{A}, \mathscr{F}_{A}(C)=C$, где $C$ - унитарный оператор, и пусть хотя бы один из операторов $A_{11}$ и $A_{22}$ унитарен. Тогда $A_{22}=\alpha C A_{11} C^{-1}$, где $\alpha \in(0,1]$.

Доказательство получается с помощью теоремы 4.

Из теорем 2, 4 и 5 и замечания 3 настоящей заметки с помощью предложений из [3] получаются наши основные утверждения:

Теорема 6. Пусть $\mathscr{F}_{A}: \mathscr{K} \rightarrow \mathscr{K}-$ афбфинное д.л.о. вида (3), где $K \in \mathscr{K} u$ либо $A_{11}=I$ и $C$-коизометрия, либо $A_{22}=I$ и $C$ - изометрия.

Тогда отображсние $\mathscr{F}_{A}$ обладает KE-свойством.

Теорема 7. Всякое отображсение $\mathscr{F}_{A}: \mathscr{K} \rightarrow \mathscr{K}$ вида (4) обладает KEсвойством.

Теорема 8. Пусть верхний треуголъный плюс-оператор $A$, такой, что $0 \in \rho\left(A_{11}\right)$, определяет на некотором подмножсестве пространства $\mathscr{L}\left(\mathfrak{H}_{1}, \mathfrak{H}_{2}\right)$ д.л.о. $\mathscr{F}_{A}, \mathscr{F}_{A}(C)=C$, где $C$ - унитарный оператор, и пусть хотя бъ один из операторов $A_{11}$ и $A_{22}$ единичный. Тогда $\left.\mathscr{F}_{A}\right|_{\mathscr{K}}$ отображсает $\mathscr{K}$ в себя и обладает KЕ-свойством. 
Ключевым моментом доказательств является теорема 2 настоящей заметки. Так, в условиях теоремы 6 из теоремы 2 следует, что в обоих случаях мы приходим к нижним треугольным операторным матрицам (плюс-операторов $A$ ), на главных диагоналях которых стоят, в некотором порядке, единичный и равномерно положительный операторы. Отсюда, согласно леммам 2 и 3 из [3], следует, что отображения $\mathscr{F}_{A}$ обладают KЕ-свойством.

\title{
ЛитерАтУРА
}

[1] V. Khatskevich, S. Reich, D. Shoikhet, Nonlinear Anal., 47:6 (2001), 3977-3988. [2] V. Khatskevich, S. Reich, D. Shoikhet, Acta Sci. Math. (Szeged), 69:1-2 (2003), 67-98. [3] В. Хацкевич, В. Сендеров, ДАН, 403:5 (2005), 607-609. [4] M. Elin, V. Khatskevich, in: Contemp. Math., vol. 382, Amer. Math. Soc., Providence, RI, 2005, 113-120. [5] M. Elin, V. Khatskevich, J. Nonlinear Convex Anal., 6:1 (2005), 173-185. [6] Н. Данфорд, Дж. Шварц, Линейные операторы, m. 1, Общая теория, Мир, М., 1962. [7] Т. Я. Азизов, И. С. Иохвидов, Основы теории линейных операторов в пространствах с индефинитной метрикой, Наука, М., 1986. [8] В. А. Хацкевич, В. А. Сендеров, Докл. РАН, 379:4 (2001), 455-458. [9] V. Khatskevich, V. Senderov, Funct. Different. Equ., 10:1/2 (2003), 239-258. [10] V. Khatskevich, V. Senderov, in: Fields Inst. Commun., vol. 25, Amer. Math. Soc., Providence, RI, 2000, 331-344. [11] P. R. Halmos, A Hilbert Space Problem Book, Van Nostrand Co., Princeton, NJToronto, Ont.-London, 1967.

email: jsadovskaya@mail.ru International College of Technology, ORT Braude

Поступило в редакцию e-mail: victor_kh@hotmail.com

22 мая 2008 г.

\section{УДК 517.9}

\section{Асимптотика интегрального ядра и регуляризованный след диффузионной полугруппы}

\author{
(C) 2010. С. А. Степин
}

Представление диффузионного ядра $p_{V}(x, y, t)$ - фундаментального решения уравнения $\partial_{t} u=(\Delta / 2+V(x)) u-$ в виде континуального (винеровского) интеграла дает способ нахождения коэффициентов асимптотического разложения

$$
p_{V}(x, y, t) \sim p_{0}(x, y, t)\left\{1+\sum_{n=1}^{\infty} c_{n}(x, y) t^{n}\right\}, \quad t \downarrow 0 .
$$

Процедура их вычисления сводится к интегрированию с весом потенциала $V$ и его частных производных по отрезку, соединяющему $x$ и $y$, и подсчету моментов $E_{\kappa}\left(s_{1}, \ldots, s_{m}\right)$ гауссовской меры вида

$$
(2 \pi)^{-m / 2}\left(\operatorname{det} a_{i j}\right)^{-1 / 2} \int_{\mathbb{R}} \cdots \int_{\mathbb{R}} x_{1}^{\kappa_{1}} \cdots x_{m}^{\kappa_{m}} \exp \left(-\frac{1}{2} a^{i j} x_{i} x_{j}\right) d x_{1} \cdots d x_{m},
$$

где $\kappa=\left(\kappa_{1}, \ldots, \kappa_{m}\right)$ - мультииндекс, $a_{i j}=\min \left\{s_{i}, s_{j}\right\}-s_{i} s_{j}$ и $a^{i j}-$ элементы матрицы, обратной к $a_{i j}$. Интегральное представление ядра $p_{V}$ применяется 